\title{
Teleorientação com o uso de ferramentas digitais no auxílio ao atendimento odontológico em época de pandemia da COVID-19: revisão integrativa da literatura
}

Teleorientation with the use of digital tools to assist dental care in a time of the COVID-19

pandemic: an integrative literature review

Teleorientación con el uso de herramientas digitales para ayudar al cuidado dental en tiempos de la pandemia COVID-19: una revisión integradora de la literatura

\section{Resumo}

No âmbito da prestação de serviços de saúde, o consultório odontológico passou por uma verdadeira revolução tecnológica, a partir da pandemia da COVID-19 para que as consultas odontológicas pudessem ser mantidas com o maior nível de segurança para profissionais e pacientes, e essa revolução passa pelo uso constante das ferramentas e recursos digitais. O objetivo desse trabalho foi identificar de que forma as ferramentas digitais podem auxiliar no atendimento odontológico no momento da pandemia da COVID-19. Foi realizada uma revisão integrativa da literatura, por meio da qual foram selecionados a partir dos critérios de elegibilidade propostos na estratégia PRISMAScR, 9 artigos para análise qualitativa, nas bases de dados Scielo e Pubmed. Percebeu-se que as ferramentas mais utilizadas são aquelas de mensagens instantâneas, como Whatsapp e Telegram, além do uso das chamadas telefônicas para o telemonitoramento e a teleconsulta, e estas ações foram fomentadas pelo reconhecimento por parte do Conselho Federal de Odontologia do uso da Teleodontologia como estratégia necessária para a manutenção dos atendimentos odontológicos. Conclui-se, portanto, que a transformação digital ocasionada pela pandemia da COVID19 deverá se tornar perene ainda que a situação epidemiológica se torne mais controlada, propiciando uma melhor comunicação entre o cirurgião dentista e seus pacientes.

Palavras-chave: Tecnologia da informação; Assistência odontológica; COVID-19.

\begin{abstract}
In the scope of the provision of health services, the dental office has undergone a true technological revolution, starting with the COVID-19 pandemic so that dental consultations could be maintained with the highest level of safety for professionals and patients, and this revolution passes for the constant use of digital tools and resources. The objective of this work was to identify how digital tools can assist in dental care at the time of the COVID-19 pandemic. An integrative literature review was conducted, through which 9 articles for qualitative analysis were selected from the eligibility criteria proposed in the PRISMA-ScR strategy, in the Scielo and Pubmed databases. It
\end{abstract}


was noticed that the most used tools are those of instant messaging, such as Whatsapp and Telegram, and phone calls for telemonitoring and teleconsultation, and these actions were fostered by the recognition by the Federal Council of Dentistry of the use of Teleodontology as a necessary strategy for maintenance dental care. It is concluded, therefore, that the digital transformation caused by the pandemic of COVID-19 should become perennial even if the epidemiological situation becomes more controlled, providing better communication between the dentist and his patients.

Keywords: Information technology; Dental care; COVID-19.

\section{Resumen}

En el ámbito de la prestación de servicios de salud, el consultorio odontológico ha experimentado una verdadera revolución tecnológica, comenzando con la pandemia COVID-19 para que las consultas dentales se pudieran mantener con el más alto nivel de seguridad para profesionales y pacientes, y esta revolución pasa por el uso constante de herramientas y recursos digitales. El objetivo de este trabajo fue identificar cómo las herramientas digitales pueden ayudar en el cuidado dental en el momento de la pandemia de COVID-19. Se realizó una revisión integradora de la literatura, mediante la cual se seleccionaron 9 artículos para análisis cualitativo de los criterios de elegibilidad propuestos en la estrategia PRISMA-ScR, en las bases de datos Scielo y Pubmed. Se notó que las herramientas más utilizadas son las de mensajería instantánea, como Whatsapp y Telegram, y llamadas telefonicas para telemonitorización y teleconsulta, y estas acciones fueron impulsadas por el reconocimiento por parte del Consejo Federal de Odontología del uso de la Teleodontología como estrategia necesaria para el mantenimiento del cuidado dental. Se concluye, por tanto, que la transformación digital provocada por la pandemia de COVID-19 debe volverse perenne aunque la situación epidemiológica se vuelva más controlada, proporcionando una mejor comunicación entre el odontólogo y sus pacientes.

Palabras-clave: Tecnología de la información; Atención odontológica ; COVID-19.

\section{Introdução}

O mundo enfrenta uma crise de saúde pública após a disseminação do vírus SARS-CoV-2 que provoca uma doença com manifestações predominantemente respiratórias (Souza et al., 2021), que pode também afetar outros sistemas do corpo humano (Souza et al., 2021; Iser et al., 2020), variando de quadros leves a quadros graves, potencialmente fatais (Amorim Dos Santos., 2021; Brandão et al., 2021). Sabe-se que o novo coronavírus é altamente transmissível o que implica diretamente na redução da procura por atendimento odontológico. Assim, é necessário buscar novas estratégias de orientação, atendimento e educação em saúde bucal para a população, em tempos de pandemia. (Ghai, 2020).

Com a pandemia da COVID-19 a população negligenciou os cuidados com a saúde, em especial a bucal, por motivos econômicos e epidemiológicos, onde os mesmos ficaram receosos com as consultas presenciais, uma vez que é potencial fonte de contaminação. Algo muito importante, no entanto, de desconhecimento de alguns pacientes, é que as patologias bucais se não tratadas corretamente podem repercutir diretamente sobre a condição geral de saúde do indivíduo (Brasil., 2020). Alguns estudos mostram que a presença de lesões orais precede os sintomas respiratórios da COVID-19 (Carvalho et al., 2021; Brandão et al., 2021). O atendimento durante o período pandêmico concentra principalmente as prioridades odontológicas. Com o aumento dos casos e consequente restrição nos atendimentos por parte do Ministério da Saúde, os atendimentos passaram a ser feitos em caráter de urgência e emergência, desde que o profissional utilize os equipamentos de proteção individual (EPI) e ocorra uma anamnese criteriosa antes do atendimento, incluindo a aferição da temperatura ainda na chegada do paciente à sala de espera (Carletto \& Santos, 2020). Cabe então ao cirurgião dentista organizar o acesso aos atendimentos de urgência/emergência e os eletivos, que são liberados quando ocorre uma melhora nos parâmetros epidemiológicos locais, adequando-se aos novos protocolos de atendimento, triagem e monitoramento, este último podendo ser feito por meio de ferramentas remotas como o atendimento pré-clínico por telefone/virtual (Brasil., 2020).

Para o serviço odontológico durante a pandemia, o cirurgião dentista deve estar atento às atualizações de biossegurança e a utilização de protocolos específicos para não haver risco de infecção cruzada e disseminação do vírus (Carletto \& Santos, 2020; Cabrera-Tasayco et al., 2020; Izzetti et al., 2020). Durante esse cenário de pandemia, o profissional deve se atentar também às condições epidemiológicas do município, de saúde do paciente, aos protocolos de proteção 
individual, de desinfecção de ambientes, entre outros (Brasil., 2020). No monitoramento digital, a análise do risco é de suma importância e pode esclarecer pontos importantes para o atendimento ou adiamento da consulta odontológica, uma vez que é possível obter informações sobre o paciente em relação às queixas ou sintomas respiratórios característicos do COVID-19, como tosse seca, falta de ar, coriza, febre, perda ou distorção de paladar e ou olfato (Brasil., 2020).

Ferramentas digitais são recursos baseados no uso da rede mundial de computadores e de dispositivos fixos ou móveis que tenham a tecnologia como a base de seu uso e funcionamento, como por exemplo, o uso de computadores, celulares, aplicativos e softwares que contribuam para a quebra de barreiras da comunicação ainda que a distância (Moretto \& Dametto, 2018). A inserção das ferramentas digitais na odontologia favorece o atendimento odontológico na pandemia da COVID-19 (Ghai, 2020). Alguns aplicativos de mensagem instantâneas como WhatsApp, Telegram, Instagram, SMS, Messenger; aplicativos de vídeo-chamada como Google Meet, Skype, Facetime, ZoomPro; e até mesmo o uso de telefones, contribuem de forma significativa como facilitador de troca de informações entre os pacientes e os cirurgiões, nesta época de pandemia. (Telles-Araujo et al., 2020). Assim, a teleorientação por meio das ferramentas digitais permite que o atendimento odontológico não tenha início na chegada do paciente ao consultório, possibilitando orientações sobre educação em saúde bucal, triagem dos pacientes e encaminhamentos, o que evita o contato físico desnecessário, reduzindo assim os riscos de contaminação (Park et al., 2021). O Conselho Federal de Odontologia (CFO) aprova o uso das ferramentas digitais para atividades de telemonitoramento e teleorientação, no entanto, não permite o exercício da odontologia a distância no que diz respeito a diagnóstico bucal e prescrição e elaboração de um plano de tratamento (CFO, 2020).

O objetivo desse estudo é identificar de que forma a teleorientação por meio de ferramentas digitais pode auxiliar no atendimento odontológico em época de pandemia da COVID-19.

\section{Metodologia}

Trata-se de um estudo de revisão integrativa da literatura em que a pergunta do estudo foi elaborada utilizando a estratégia PICO (Santos et al., 2007) em que atribui-se P (população) aos pacientes que buscam atendimento odontológico na pandemia de COVID-19, I (indicador) ao uso das ferramentas digitais auxiliando no atendimento odontológico durante a pandemia, C (controle) ao atendimento odontológico na pandemia sem o auxílio das ferramentas digitais e O (desfecho) se o uso das ferramentas digitais contribuem positivamente para o atendimento odontológico frente a pandemia da COVID-19.

A pergunta norteadora do estudo é: Como as ferramentas digitais auxiliam no atendimento aos pacientes que buscam atendimento odontológico, em época de pandemia de COVID-19?

Esta revisão integrativa foi relatada de acordo com o Guia PRISMA ScR (Principais Itens para Relatar Revisões sistemáticas e Meta-análises) (Galvão et al., 2015). Os artigos foram buscados na base de dados Pubmed/Medline e Google Scholar. As palavras de busca utilizadas foram "technology", "dental care" e "COVID-19" acrescido do operador boleano “and". Os critérios de inclusão dos artigos na pesquisa foram estudos disponíveis na íntegra e compreendidos entre os anos 2020 e 2021. Como critério de exclusão foram os artigos apenas com resumos disponíveis, opiniões de especialistas, teses e dissertações, bem como, artigos em idiomas diversos ao inglês e ao português.

\section{Resultados}

A busca de dados resultou na identificação de 66 artigos potencialmente elegíveis, dos quais 58 encontrados na base de dados PubMed e 8 na base de dados Google Scholar por meio da ferramenta de busca que direciona para base da Scielo. Não foram utilizadas outras fontes de publicações, conforme demonstrado na Figura 1. 
Figura 1 - Fluxograma do processo de seleção dos estudos primários.
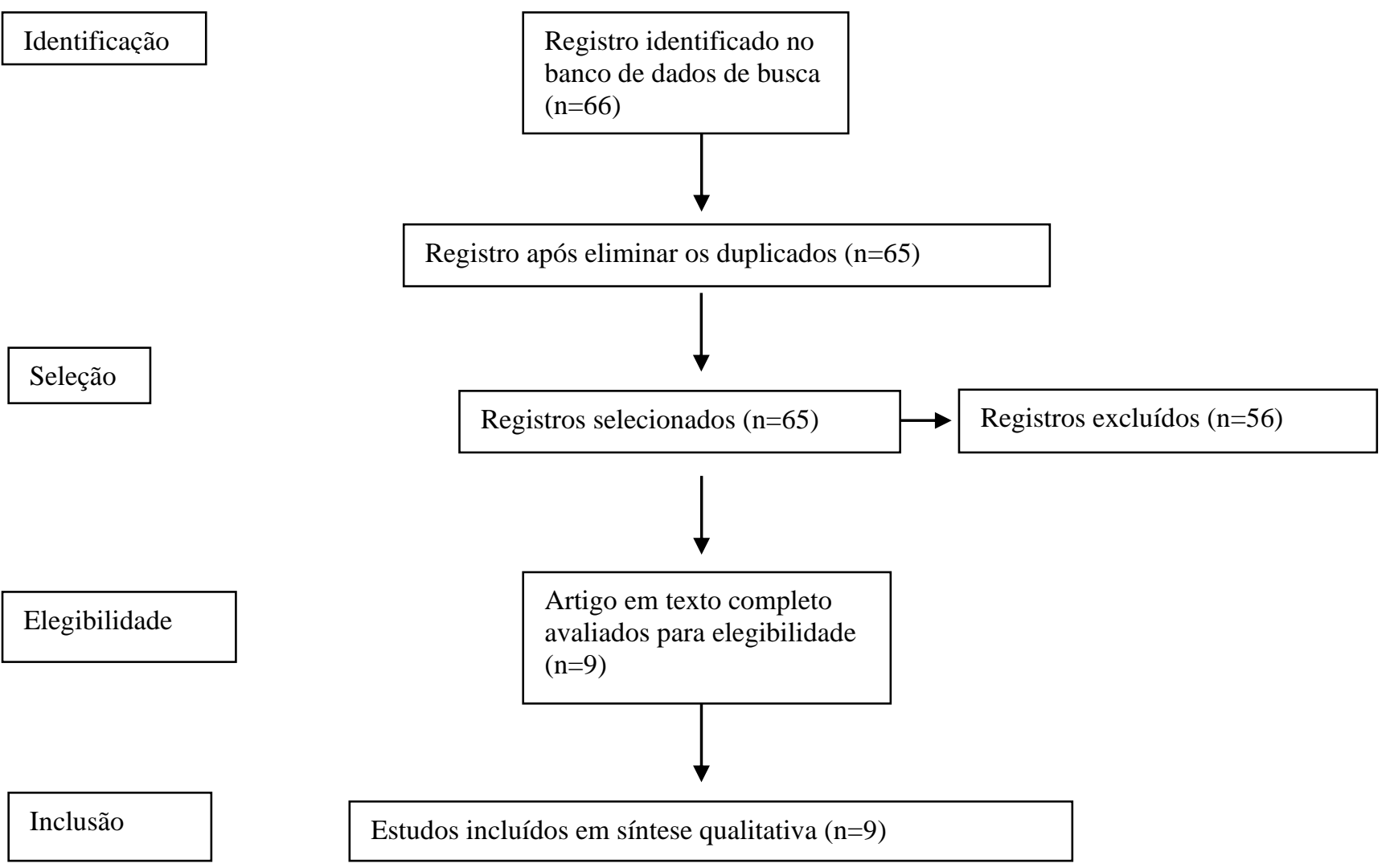

Fonte: Autores.

Apenas 1 artigo foi eliminado, pois estava presente em ambas as bases de dado sendo considerado dessa forma duplicado. A leitura dos títulos e dos resumos dos artigos foram os métodos utilizados para inclusão e exclusão, tendo sido eliminados 56 publicações. Ao final da análise alcançou-se a amostra final de 9 estudos primários. Os artigos excluídos não correspondiam durante a leitura de seu título ou resumo aos desfechos esperados nesta revisão, ou não se relacionavam ao escopo central dessa pesquisa.

Após elegibilidade dos artigos, os mesmos foram organizados em um quadro (Quadro 1) baseada na ferramenta MMAT (Galvão \& Ricarte, 2019), com a descrição do autor, ano de publicação, periódico, tipo de estudo, resultado e ferramenta digital utilizada. 
Quadro 1 - Caracterização do estudo, segundo autor principal, ano de publicação, periódico, objetivos, principais resultados e ferramentas digitais utilizadas.

\begin{tabular}{|c|c|c|c|c|}
\hline Autor/ Ano & Periódico & Objetivo & Resultados & Ferramentas Digitais \\
\hline Ghai, 2020 & $\begin{array}{l}\text { Diabetes \& } \\
\text { metabolic } \\
\text { syndrome }\end{array}$ & $\begin{array}{l}\text { Teleodontoglogia como solução } \\
\text { inovadora para retomada da } \\
\text { prática clínica odontológica } \\
\text { durante a pandemia }\end{array}$ & $\begin{array}{l}\text { Facilitação remota do tratamento, } \\
\text { educação e orientação por meio das } \\
\text { ferramentas tecnológicas. } \\
\text { Teleconsulta, telediagnóstico, } \\
\text { teletriagem e telemonitoramento }\end{array}$ & $\begin{array}{l}\text { Teletriagem via } \\
\text { smartphone, por meio de } \\
\text { chamadas telefônicas e } \\
\text { via WhatsApp }\end{array}$ \\
\hline $\begin{array}{l}\text { Telles- } \\
\text { Araujo et } \\
\text { al., } 2020\end{array}$ & Clinics & $\begin{array}{l}\text { A teleorientação permite aos } \\
\text { profissionais realizarem } \\
\text { triagem, orientação e } \\
\text { encaminhamento do paciente de } \\
\text { forma isolada. Além de permitir } \\
\text { o monitoramento visual de } \\
\text { lesões bucais por meio de } \\
\text { fotografias. Apoiar o SUS } \\
\text { durante a pandemia. }\end{array}$ & $\begin{array}{l}\text { Permitir ao profissional } \\
\text { monitoramento visual, troca de } \\
\text { informações entre profissionais, } \\
\text { suporte diagnóstico }\end{array}$ & $\begin{array}{l}\text { Aplicativos de mensagens } \\
\text { instantâneas (WhatsApp, } \\
\text { Telegram, Instagram, } \\
\text { SMS, Messenger) e } \\
\text { aplicativos de } \\
\text { videochamada (Google } \\
\text { Meet, Skype, Facetime, } \\
\text { WhatsApp). }\end{array}$ \\
\hline $\begin{array}{l}\text { Talla et al., } \\
2020\end{array}$ & $\begin{array}{l}\text { Quintessence } \\
\text { International }\end{array}$ & $\begin{array}{l}\text { Revisar conhecimento do uso } \\
\text { da teleodontologia antes da } \\
\text { pandemia; Como a } \\
\text { teleodontologia pode ser usada } \\
\text { no momento da pandemia; } \\
\text { demonstrar as intervenções que } \\
\text { a teleodontolodia é e não é } \\
\text { eficaz e com a relação custo } \\
\text { benefício. }\end{array}$ & $\begin{array}{l}\text { Fornecer conhecimento e propor } \\
\text { orientações para o uso da } \\
\text { teleodontolgia para atendimento } \\
\text { odontológico no contexto da } \\
\text { COVID-19 }\end{array}$ & $\begin{array}{l}\text { Telefone, texto ou e-mail, } \\
\text { videoconferência ( } \\
\text { ZoomPro, Microsoft } \\
\text { Teams ou Webex). }\end{array}$ \\
\hline $\begin{array}{l}\text { Mascitti \& } \\
\text { Campisi, } \\
2020\end{array}$ & $\begin{array}{l}\text { International } \\
\text { Journal of } \\
\text { Environmental } \\
\text { Research and } \\
\text { Public Health }\end{array}$ & $\begin{array}{l}\text { O uso das ferramentas digitais } \\
\text { na prevenção de saúde bucal, } \\
\text { desafios e questões atuais a } \\
\text { cerca deste tema. }\end{array}$ & $\begin{array}{l}\text { O uso da teleodontologia para } \\
\text { manejo remoto do paciente. No } \\
\text { entanto, isso esbarra em alguns } \\
\text { desafios como a falta de } \\
\text { alfabetização digital, exclusão } \\
\text { digital, privacidade do paciente, } \\
\text { adequação aos regulamentos } \\
\text { internacionais, conflitos e mal } \\
\text { entendimento entre paciente e } \\
\text { profissional }\end{array}$ & $\begin{array}{l}\text { Tecnologia da informação } \\
\text { e comunicação e } \\
\text { dispositivos móveis. }\end{array}$ \\
\hline $\begin{array}{l}\text { Carrer et } \\
\text { al., } 2020\end{array}$ & $\begin{array}{l}\text { Pesquisa } \\
\text { Brasileira em } \\
\text { Odontopediatria e } \\
\text { Clínica Integrada }\end{array}$ & $\begin{array}{l}\text { Teleodontologia como } \\
\text { alternativa para abranger e } \\
\text { qualificar os cuidados em saúde } \\
\text { bucal no SUS }\end{array}$ & $\begin{array}{l}\text { Uso das tecnologias de informação } \\
\text { e comunicação no atendimento } \\
\text { odontológico remoto por meio da } \\
\text { teleodontologia, telemonitoramento, } \\
\text { e teleorientação. Definições e } \\
\text { possibilidade de registro e atuação } \\
\text { na atenção primária. }\end{array}$ & $\begin{array}{l}\text { Consultas e triagem por } \\
\text { telefone e vídeo, } \\
\text { WhatsApp. }\end{array}$ \\
\hline $\begin{array}{l}\text { Dos Santos } \\
\& \text { Barbosa, } \\
2020\end{array}$ & Brazilian Journals & $\begin{array}{l}\text { Orientar os dentistas a cerca das } \\
\text { medidas preventivas, } \\
\text { atendimento de urgências e } \\
\text { emergências na pandemia com } \\
\text { o intuito de reduzir o risco de } \\
\text { contaminação }\end{array}$ & $\begin{array}{l}\text { Panorama do atendimento } \\
\text { odontológico em tempos de } \\
\text { COVID-19, recomendações de } \\
\text { acordo com as normas de cada país, } \\
\text { regulamentações para os serviços de } \\
\text { telessaúde. Utilização de testes } \\
\text { rápidos e aferição da temperatura. }\end{array}$ & $\begin{array}{l}\text { Consultas e triagem por } \\
\text { telefone e vídeo, } \\
\text { WhatsApp, assistência } \\
\text { virtual. }\end{array}$ \\
\hline $\begin{array}{l}\text { Santana et } \\
\text { al., } 2020\end{array}$ & $\begin{array}{l}\text { Revista Brasileira } \\
\text { de Epidemiologia }\end{array}$ & $\begin{array}{l}\text { Teleodontologia como recurso } \\
\text { de assistência odontológica } \\
\text { durante a pandemia de COVID- } \\
19\end{array}$ & $\begin{array}{l}\text { As estratégias de telecomunicação } \\
\text { são adequadas para o cenário atual } \\
\text { da pandemia. }\end{array}$ & $\begin{array}{l}\text { Chamadas de voz e vídeo; } \\
\text { WhatsApp }\end{array}$ \\
\hline
\end{tabular}




\begin{tabular}{|l|l|l|l|l|}
\hline $\begin{array}{l}\text { Aquilanti et } \\
\text { al., 2020 }\end{array}$ & $\begin{array}{l}\text { International } \\
\text { Journal of } \\
\text { Environmental } \\
\text { Research and } \\
\text { Public Health }\end{array}$ & $\begin{array}{l}\text { Avaliar o impacto da pandemia } \\
\text { na procura por atendimento } \\
\text { odontológico na Itália. }\end{array}$ & $\begin{array}{l}\text { Mais estudos são necessários para } \\
\text { avaliar o impacto da pandemia na } \\
\text { saúde bucal o o impacto da } \\
\text { pandemia no atendimento } \\
\text { odontológico. }\end{array}$ & $\begin{array}{l}\text { E-mail, chamadas } \\
\text { telefônicas, redes sociais, } \\
\text { mensagens. }\end{array}$ \\
\hline $\begin{array}{l}\text { Park et al., } \\
2021\end{array}$ & $\begin{array}{l}\text { Teledentistry } \\
\text { Platforms of } \\
\text { Orthodontics }\end{array}$ & $\begin{array}{l}\text { Fornecer informações aos } \\
\text { ortodontistas que desejam } \\
\text { realizar a prática remota. }\end{array}$ & $\begin{array}{l}\text { Ressalta os avanços da tecnologia, a } \\
\text { realização das consultas virtuais e } \\
\text { as plataformas disponíveis para o } \\
\text { atendimento }\end{array}$ & $\begin{array}{l}\text { Zoom, Google, Skype, } \\
\text { Smile Virtual, Review } \\
\text { Tools, Smile Snap } \\
\text { Rhinogram, Carestack, } \\
\text { TeleDent Teledentix }\end{array}$ \\
\hline
\end{tabular}

Fonte: Autores.

Percebe-se com os resultados obtidos que as ferramentas digitais são úteis para a manutenção dos atendimentos odontológicos, bem como existe uma gama de ferramentas digitais que podem ser utilizadas, nas quais destaca-se o WhatsApp como recurso de comunicação entre cirurgiões dentistas e seus pacientes. As chamadas telefônicas também se destacam como forma de teleorientação sendo a forma mais clássica de se obter um contato prévio ao atendimento.

\section{Discussão}

Após análise dos 9 estudos primários notou-se que o uso das ferramentas digitais é realidade em uma prestação de serviços odontológicos em tempos de pandemia, pois além de facilitar a interação profissional paciente, é um método muito útil que contribui para o atendimento odontológico de forma segura sem o risco de transmissão comunitária da COVID-19. Para realizar o atendimento a distância, várias ferramentas estão disponíveis, dentre elas os smartphones, com o uso de aplicativos de mensagens instantâneas como o WhatsApp (Telles-Araujo et al., 2020; Carrer et al., 2020; Dos Santos \& Barbosa, 2020; Santana et al., 2020; Telegram, Instagram, SMS e Messenger (Telles-Araujo et al., 2020; Aquilanti et al., 2020). Outras ferramentas também disponíveis, são os aplicativos para vídeo chamada como WhatsApp (Telles-Araujo et al., 2020; Carrer et al., 2020; Dos Santos \& Barbosa, 2020; Santana et al., 2020), Google Meet, Skype, Facetime (Telles-Araujo et al., 2020), ZoomPro, MicrosoftTeams e Webex (Talla et al., 2020). Esta comunicação pode ser feita também, por meio de chamadas telefônicas (Ghai, 2020; Talla et al., 2020; Carrer et al., 2020; Dos Santos \& Barbosa, 2020; Santana et al., 2020; Aquilanti et al., 2020) e e-mails (Talla et al., 2020; Aquilanti et al., 2020).

As estratégias de telecomunicação são pertinentes para o cenário global pandêmico e tende a se popularizar com o tempo, integrando-se ao atendimento odontológico e mantendo-se mesmo após passado a pandemia da COVID-19 (Santana et al., 2020). A teleconsulta é o termo utilizado para a realização de consultas virtuais, que pode ser realizada de forma síncrona, quando a interação mesmo que online no mesmo tempo e espaço para profissional e paciente, como por exemplo em uma chamada de vídeo ou assíncrona que corresponde por exemplo à comunicação por mensagem no Whatsapp ou email, na qual o canal de comunicação está aberto, no entanto as respostas não necessariamente são imediatas (Carrer et al., 2020). Já o telemonitoramento, é o monitoramento remoto do paciente, que permite realizar coleta de dados clínicos e acompanhamento de procedimentos cirúrgicos e não cirúrgicos, e resulta em redução reduz custos e tempo de espera (Carrer et al., 2020; Ghai, 2020). A teleorientação, permite a realização da fase inicial de triagem, o possibilita a indicação do momento ideal para o atendimento presencial, bem como a realização de atividades educativas e de esclarecimentos. Já essas duas últimas modalidades, são autorizadas pelo CFO, para serem realizadas pelo cirurgião dentista (CFO, 2020).

A respeito do uso das ferramentas digitais na odontologia elas podem ser empregadas em ações como: facilitação remota do tratamento, educação e orientação (Ghai., 2020) teleconsulta, teletriagem, telemonitoramento (Carrer et al., 2020); ( Ghai., 2020) troca de informações entre profissionais, suporte diagnóstico (Telles-Araujo et al.,2020); redução do risco de 
contaminação (Dos Santos \& Barbosa, 2020) entre outros. Entre os pontos positivos que sustentam a importância do uso das ferramentas digitais se destacam o uso em educação e saúde bucal, atenção em saúde de áreas remotas, teletriagem, redução de filas de espera, de contaminação e afins (Santana et al., 2020). No entanto, as ferramentas digitais também apresentam limitações como àquelas relativas à condição socioeconômica do paciente, já que nem todos conseguem ter acesso aos recursos tecnológicos e à internet. Outro ponto, é a falta de familiaridade que alguns pacientes possuem com tais ferramentas sendo também um fator limitador do atendimento odontológico à distância (Talla et al., 2020). No entanto, vale salientar que esta limitação no manuseio destas ferramentas vem ao longo do tempo sendo cada vez mais superadas mesmo naquelas faixas de idade mais avançadas. Vale ressaltar também, que o CFO aprova o uso das ferramentas digitais para atividades de telemonitoramento e teleorientação, porém não autoriza o exercício da odontologia a distância no que tange ao diagnóstico bucal, e a elaboração e execução de planos de tratamento (Carrer et al., 2020).

Outro ponto importante diz respeito à transmissibilidade do vírus e o papel do cirurgião dentista na prevenção da COVID -19 (Dos Santos \& Barbosa, 2020). Com a utilização das ferramentas digitais, é possível reduzir a quantidade de pacientes em filas de espera, classifica-los por meio de teletriagem (Santana et al., 2020), identificar os pacientes com suspeita de estarem infectados pelo vírus Sars-Cov-2, determinar a necessidade de atendimento e tratamento imediato ou tardio e com isso reduzindo de forma significativa a transmissão e o contágio entre pacientes e profissionais (Mascitti \& Campisi 2020). No entanto, apesar destas medidas serem demasiadamente importantes, é possível que o paciente, mesmo assintomático, esteja contaminado com o vírus e desta forma ainda ser potencial transmissor, apesar deste ainda ser um tema controverso dado o conhecimento ainda novo sobre a COVID-19.

Vale ressaltar que para o atendimento presencial, o cirurgião dentista deve se atentar aos protocolos de biossegurança disponíveis e indicados para a prevenção da transmissibilidade e contaminação da COVID-19. Vale salientar também que por se tratar de uma profissão de alto risco de transmissibilidade e contaminação, não apenas da COVID-19, mas de tantas outras patologias passiveis de contaminação por meio do campo de trabalho do Cirurgião-Dentista, que é a boca, estes profissionais são bem capacitados durante o seu curso de graduação, independente da instituição de formação. Há um treinamento e conscientização bastante extensiva, onde são orientados e cobrados rotineiramente a fazer o uso praticamente das mesmas proteções orientadas para o COVID-19, como EPI's, barreiras protetivas, óculos de proteção, máscaras, luvas, protetores faciais, e roupas adequadas para o ambiente clínico/hospitalar (Dos Santos \& Barbosa, 2020), já que a inalação de aerossol, gotículas de saliva, contato direto com as membranas mucosas, instrumentos e superfícies contaminadas aumenta o risco de transmissão do vírus durante os procedimentos odontológicos. (Aquilanti et al., 2020).

A tecnologia transformou diversos aspectos da vida social, onde as ferramentas digitais juntamente com os equipamentos eletrônicos necessários para seu uso, facilitaram a troca de informações entre profissionais e pacientes principalmente nesta época de pandemia da COVID-19. No entanto, as plataformas utilizadas não são apenas ideias para atendimento em tempos de pandemia, reduzindo o contágio da doença, e sim para aumentar a ligação entre profissional e paciente, derrubando barreiras e favorecendo a manutenção do autocuidado apoiado mesmo em tempos de atendimento presencial, sendo uma herança positiva da COVID-19, para a Odontologia (Park et al., 2021).

\section{Conclusão}

Após análise da literatura observou-se que o uso das ferramentas digitais para o atendimento odontológico tem se tornado comum na prestação de serviços odontológicos, especialmente no que tange a um período de pandemia. De acordo com esta pesquisa, a ferramenta mais utilizada foi o WhatsApp na qual é possível realizar o envio de mensagens, chamadas de voz e vídeo. Percebe-se ainda que a chamada telefônica que também pode se utilizar de recursos digitais como a telefonia móvel não perdeu espaço quando no contexto da teleorientação ou prévio ao atendimento odontológico. A teleodontologia 
tornou-se uma solução para continuidade da prática odontológica durante o período pandêmico e tende a ser um método que continuará sendo utilizado mesmo após a finalização deste período.

Mais estudos são necessários nessa temática, especialmente no que tange à manutenção dos cuidados em saúde bucal, abordando a adesão dos pacientes ao uso das ferramentas digitais e o impacto econômico do uso dessas ferramentas para o consultório odontológico, do ponto de vista da fidelização dos clientes.

\section{Referências}

Aquilanti, L., Gallegati, S., Temperini, V., Ferrante, L., Skrami, E., Procaccini, M., \& Rappelli, G. (2020). Italian Response to Coronavirus Pandemic in Dental Care Access: The DeCADE Study. International journal of environmental research and public health, 17(19), 6977. https://doi.org/10.3390/ijerph17196977

Amorim Dos Santos, J., Normando, A., Carvalho da Silva, R. L., Acevedo, A. C., De Luca Canto, G., Sugaya, N., Santos-Silva, A. R., \& Guerra, E. (2021). Oral Manifestations in Patients with COVID-19: A Living Systematic Review. Journal of Dental Research, 100(2), 141-154. https://doi.org/10.1177/0022034520957289

Brandão, T. B., Gueiros, L. A., Melo, T. S., Prado-Ribeiro, A. C., Nesrallah, A., Prado, G., Santos-Silva, A. R., \& Migliorati, C. A. (2021). Oral lesions in patients with SARS-CoV-2 infection: could the oral cavity be a target organ?. Oral surgery, oral medicine, oral pathology and oral radiology, 131(2), e45e51. https://doi.org/10.1016/j.oooo.2020.07.014

Brasil. (2020). Guia de orientação para atenção odontológica no contexto da COVID-19. Ministério da Saúde. Brasília- DF, (1), 1-86. https://www.gov.br/saude/pt-br/media/pdf/2020/novembro/17/17_12_guia-de-orientacaoes-para-atencao-odontologica-no-contexto-da-covid-19.pdf

Cabrera-Tasayco, F., Rivera-Carhuavilca, J. M., Atoche-Socola, K. J., Peña-Soto, C., \& Arriola-Guillén, L. E. (2020). Biosafety Measures at the Dental Office After the Appearance of COVID-19: A Systematic Review. Disaster medicine and public health preparedness, 1-5. Advance online publication. https://doi.org/10.1017/dmp.2020.269

Carletto, A. F., \& Santos, F. F. D. (2020). A atuação do dentista de família na pandemia do Covid-19: o cenário do Rio de Janeiro. Physis: Revista de Saúde Coletiva, 30(3), e300310. Epub September 04, 2020.https://doi.org/10.1590/s0103-73312020300310

Carrer, F. C. D. A., Matuck, B., Lucena, Edson H. G. D., Martins, F. C., Pucca Junior, G. A., Galante, M. L., Tricoli, M. F. D. M., Macedo, M. C. S. (2020). Teleodontologia e SUS: uma importante ferramenta para a retomada da Atenção Primária à Saúde no contexto da pandemia de COVID-19. Pesquisa Brasileira em Odontopediatria e Clínica Integrada. https://doi.org/10.1590/SciELOPreprints.837

Carvalho, T. de A., Silva, D. L. M., Oliveira, L. C., Machado, F. C., Andrade, R. S. de, \& Novais, V. R. (2021). What oral cavity can show in COVID-19 patients? Literature integrative review. Research, Society and Development, 10(4), e17710414072. https://doi.org/10.33448/rsd-v10i4.14072

Conselho Federal de Odontologia. (2020). Resolução CFO-226, de 04 de Junho de 2020.Conselho Federal de Odontologia, Brasília-DF, 1-3. http://sistemas.cfo.org.br/visualizar/atos/RESOLU\%c3\%87\%c3\%83O/SEC/2020/226.

Dos Santos, K. F., Barbosa, M., (2020). COVID-19 e a Odontologia na prática atual. Brazilian Journals. 1-23. https://doi.org/10.1590/SciELOPreprints.746

Galvão, M. C. B., \& Ricarte, I. L. M. (2019). Revisão sistemática da literatura: conceituação, produção e publicação. Logeion: Filosofia Da Informação, 6(1), 57-73. https://doi.org/10.21728/logeion.2019v6n1.p57-73

Galvão, T. F., Pansani, T. S. D. A., \& Harrad, D. (2015). Principais itens para relatar Revisões sistemáticas e Meta-análises: A recomendação PRISMA. Epidemiologia e Serviços de Saúde, 24(2), 335-342. https://doi.org/10.5123/S1679-49742015000200017

Ghai S. (2020). Teledentistry during COVID-19 pandemic. Diabetes \& metabolic syndrome, 14(5), 933-935. https://doi.org/10.1016/j.dsx.2020.06.029

Iser, B. P. M., Sliva, I., Raymundo, V. T., Poleto, M. B., Schuelter-Trevisol, F., \& Bobinski, F. (2020). Definição de caso suspeito da COVID-19: uma revisão narrativa dos sinais e sintomas mais frequentes entre os casos confirmados. Epidemiologia e Serviços de Saúde, $29(3)$, e2020233. https://dx.doi.org/10.5123/s1679-49742020000300018

Izzetti, R., Nisi, M., Gabriele, M., \& Graziani, F. (2020). COVID-19 Transmission in Dental Practice: Brief Review of Preventive Measures in Italy. Journal of Dental Research, 99(9), 1030-1038. https://doi.org/10.1177/0022034520920580

Mascitti, M., \& Campisi, G. (2020). Dental Public Health Landscape: Challenges, Technological Innovation and Opportunities in the 21st Century and COVID-19 Pandemic. International journal of environmental research and public health, 17(10), 3636. https://doi.org/10.3390/ijerph17103636

Moretto, I.M. \& Dametto, J. (2018). Desafios educacionais da era digital: adversidades e possibilidades do uso da tecnologia na prática docente. Perspectiva, 42(160), 77-87.

Park, J. H., Rogowski, L., Kim, J. H., Al Shami, S., \& Howell, S. (2021). Teledentistry Platforms for Orthodontics. The Journal of clinical pediatric dentistry, 45(1), 48-53. https://doi.org/10.17796/1053-4625-45.1.9

Santana, L. A. D. M., Santos, M. A. L. D., Albuquerque, H. I. M. D., Costa, S. F. D. S., Rezende-Silva, E., Gercina, A. C., \& Takeshita, W. M. (2020). Teledentistry in Brazil: a viable alternative during COVID-19 pandemic. Revista Brasileira de Epidemiologia, 23, e200082. https://doi.org/10.1590/1980549720200082 . 
Research, Society and Development, v. 10, n. 6, e17210615663, 2021

(CC BY 4.0) | ISSN 2525-3409 | DOI: http://dx.doi.org/10.33448/rsd-v10i6.15663

Santos, C. M. C., Pimenta, C. A. M., \& Nobre, M. R. C., (2007). The PICO strategy for the research question construction and evidence search. Revista Latino-Americana de Enfermagem, 15(3), 508-511. https://doi.org/10.1590/S0104-11692007000300023

Souza, A. S. R., Amorim, M. M. R., Melo, A. S. D. O., Delgado, A. M., Florêncio, A. C. M. C. da C., Oliveira, T. V. de, Lira, L. C. S., Sales, L. M. dos S., Souza, G. A., Melo, B. C. P. de, Morais, Í., \& Katz, L. (2021). General aspects of the COVID-19 pandemic. Revista Brasileira de Saúde Materno Infantil, 21(Suppl. 1), 29-45. Epub February 24, 2021.https://doi.org/10.1590/1806-9304202100s100003

Talla, P. K., Levin, L., Glogauer, M., Cable, C., \& Allison, P. J. (2020). Delivering dental care as we emerge from the initial phase of the COVID-19 pandemic: teledentistry and face-to-face consultations in a new clinical world. Quintessence international (Berlin, Germany: 1985), 51(8), 672-677. https://doi.org/10.3290/j.qi.a44920

Telles-Araujo, G. T., Caminha, R., Kallás, M. S., \& Santos, P. (2020). Teledentistry support in COVID-19 oral care. Clinics (Sao Paulo, Brazil), 75, e2030. https://doi.org/10.6061/clinics/2020/e2030 